\title{
VISCOELASTICIDAD DE POLÍMEROS EN SOLUCIÓN ACUOSA: ESTUDIO MICROREOLÓGICO-ESTRUCTURAL
}

\author{
Viscoelasticity of polymers in solution \\ Aquosa: microreological-structural study
}

\section{EPISTEMUS}

ISSN: 2007-8196 (electrónico)

ISSN: 2007-4530 (impresa)

\section{Rogelio Gamez Corrales ${ }^{1}$ \\ Emmanuel Robles Ávila²}

Recibido: 27 de abril de 2017,

Aceptado: 22 de junio de 2017

Autor de Correspondencia:

Dr. Rogelio Gamez Corrales

Correo: rogelio@correo.fisica.uson.mx

\section{Resumen}

En este trabajo se presenta un estudio del comportamiento viscoelástico de dos sistemas de polímeros que forman geles físicos transitorios: ácido desoxirribonucleicos (ADN) disuelto en buffer y un polímero asociativo del tipo multiuniones, ambos en el régimen de concentraciones semidiluido no entrelazado. La viscoelasticidadlineal se estudió utilizando la técnica de reología mecánica, determinando los módulos elástico $G^{\prime}(\omega)$ y $G^{\prime \prime}(\omega)$ viscoso en el rango de deformaciones de bajas frecuencias y la técnica de microreología óptica para altas frecuencias. La implementación de ambas técnicas, en el estudio reológico de estos sistemas, permite extender el rango de oscilaciones de $100 \mathrm{~s}^{-1}$ a 100,000 s-1, permitiendo entender la conexión que existe entre la propiedad de viscoelasticidad de las soluciones acuosas y la estructura microscópicas de estos dos sistemas bajo deformaciones externas.

\begin{abstract}
In this paper, a study of the viscoelastic behavior of two polymer systems that form transient physical gels are presented: deoxyribonucleic acid (DNA) dissolved in buffer and an associative polymer of the multi-junction type, both in the semi-diluted non-interlaced concentrations regime. The linear viscoelasticity was studied using the mechanical rheology technique, determining the elastic $G^{\prime}(\omega)$ and $G^{\prime \prime}(\omega)$ viscous modules in the range of low frequency deformations and the optical microreology technique for high frequencies. The implementation of both techniques, in the rheological study of these systems, allows us to extend the range of oscillations from $100 \mathrm{~s}^{-1}$ to $100,000 \mathrm{~s}^{-1}$, allowing us to understand the connection between the properties of viscoelasticity of aqueous solutions and the microscopic structure. of these two systems under external deformations.
\end{abstract}

1 Departamento de Física / Correo: rogelio@correo.fisica.uson.mx

2 Departamento de Investigación en Polímeros y Materiales / Correo: emmanuel.robles@correo.fisica.uson.mx 


\section{INTRODUCCIÓN}

La reometría es una técnica muy útil para obtener información de las propiedades mecánicas de los materiales, por lo que en la industria es muy utilizada como una técnica estandarizada para la determinación de la elasticidad, viscosidad y/oviscoelasticidad. Sin embargo, el potencial de esta técnica no se limita únicamente a la determinación de las propiedades macroscópicas o de bulto de los materiales, sino que también permite entender a nivel microscópico la estructura, así como las interacciones moleculares que dan origen a las propiedades reológicas (e.g. la difusión de cadenas de polímeros). A pesar de las bondades que ofrece esta técnica en el estudio de los materiales complejos, tiene limitaciones que la hacen difícil de aplicar e interpretar. Por ejemplo, la poca cantidad de muestra que se encuentra en algunos sistemas como es el caso de materiales biológicos, o donde la estructura se destruye a deformaciones muy pequeñas. Además de necesitar gran cantidad de muestra para realizar las mediciones, también se encuentra con efectos inerciales al operar los reómetros a altas frecuencias, disminuyendo así el rango efectivo de las diferentes deformaciones aplicadas sobre la muestra.

Recientemente se han innovado técnicas experimentales con la finalidad de estudiar a nivel local microscópico el comportamiento reológico de los fluidos complejos. Éstas técnicas se basan principalmente en introducir en el sistema de estudio, partículas coloidales débilmente interactuantes con el medio circundante (Figura 1), y posteriormente realizar las mediciones del desplazamiento cuadrático medio con la finalidad de determinar la dinámica traslacional de las partículas. Así, las propiedades mecánicas de los materiales pueden ser determinadas cuantificando el desplazamiento cuadrático medio de las partículas de prueba, derivados de las fluctuaciones térmicas del medio, los desplazamientos cuadráticos medios pueden ser medidos utilizando una variedad de técnicas experimentales como: dispersión de onda difusiva (DWS), video microscopía, dispersión dinámica de luz (DLS), entre otras[1]. El rango máximo de frecuencias de microreología está limitado a la resolución temporal del instrumento utilizado en la medición de la difusión de las partículas de prueba[2]. En la Figura 2, se muestra una representación esquemática de las técnicas de reología mecánica y de microreología utilizando dispersiones de luz y sus limitantes en el rango de frecuencia. En el caso de DLS, la variación temporal de la intensidad dispersada en un medio ergódico permite obtener la difusión de las partículas trazadoras en el medio viscoelástico. Esto es, los desplazamientos cuadráticos medios $\left\langle\Delta r^{2}(t)\right\rangle$ de las partículas se obtienen a partir de las funciones de autocorrelación de intensidades $g_{2}(t)$. La técnica de dispersión dinámica de luz permite una resolución temporal y espacial, aproximadamente de $10^{-6} \mathrm{~s}$ y $10^{-1} \mathrm{~nm}$ respectivamente. Las propiedades viscoelásticas del material son obtenidas utilizando la ecuación generalizada de Stokes-Einstein asumiéndose que el medio puede ser descrito por una viscosidad compleja dependiente de la frecuencia. Donde las propiedades viscoelásticas locales pueden ser descritas utilizando los módulos elásticos $G^{\prime}(\omega)$ y viscosos $G^{\prime \prime}(\omega)$, derivados de la difusión de las partículas de prueba dispersas en el medio complejo. Originalmente esta ecuación fue formulada para coloides monodispersos en un medio puramente viscoso.
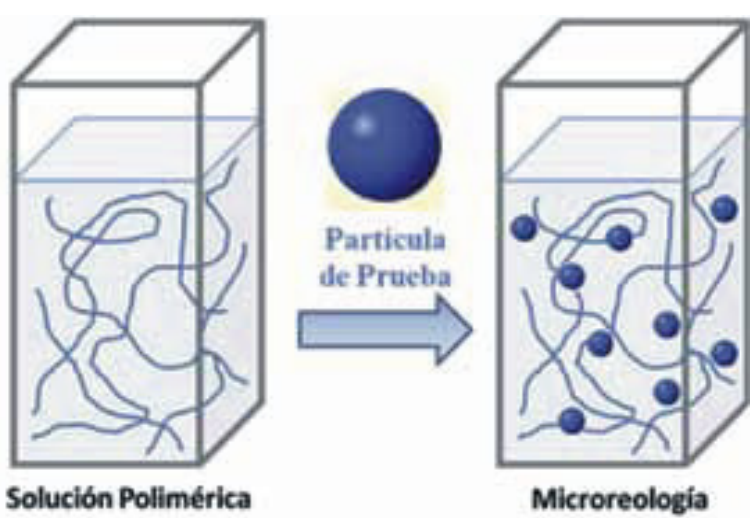

Solución Polimérica

Figura 1. Representación esquemática de los sistemas de geles físicos con y sin partículas coloidales inmersas en la solución.

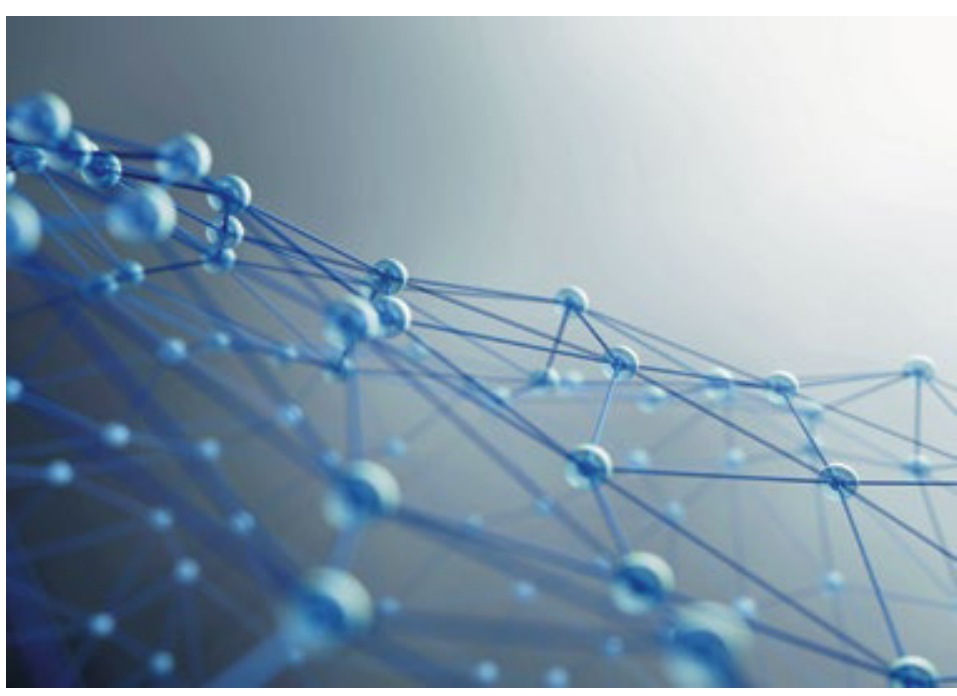

Figura 2. Representación esquemática de las técnicas de reología mecánica y microreología utilizando dispersiones de luz (DWS, DLS) y el rango de frecuencias valido. 


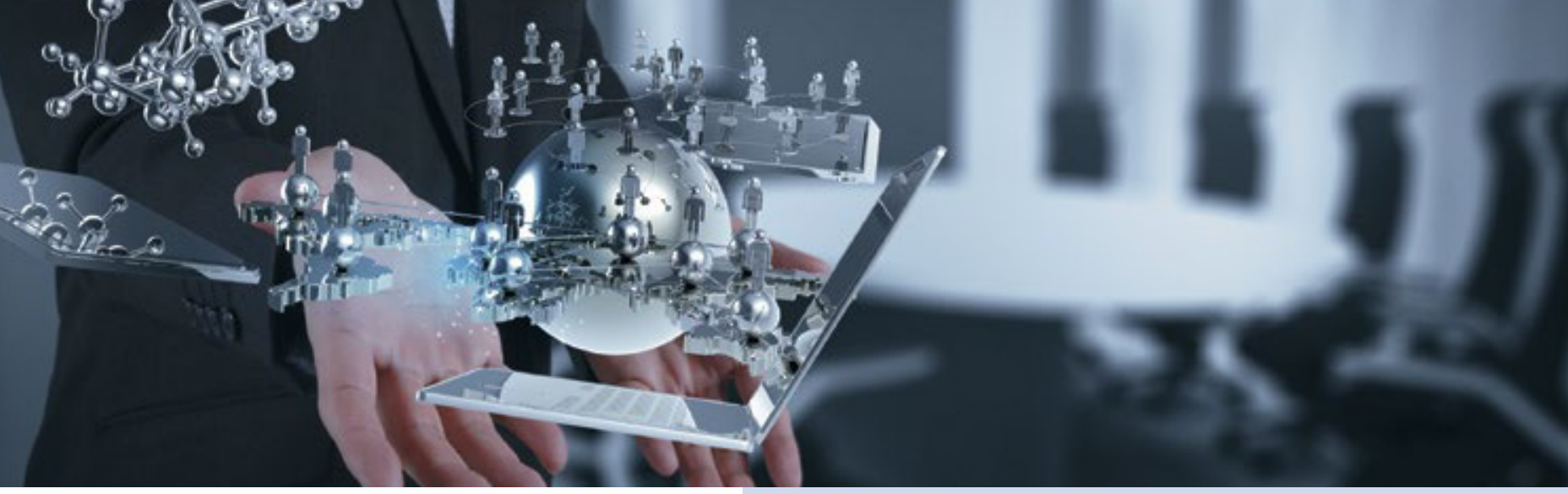

La técnica de microreología ha resultado ser una técnica experimental fundamental en la correlación de las propiedades viscoelásticas y la estructura microscópica de una gran cantidad de materiales, como: tensoactivos, polímeros, biopolímeros, polímeros asociativos [3], coloides e inclusive sistemas biológicos como el ADN [4] o los filamentos de actina. Los polímeros asociativos hidrófobamente modificados (PA) son macromoléculas compuestas por un esqueleto hidrófilo y grupos hidrófobos repartidos a ambos extremos de la cadena principal (telequélicos) y/o a lo largo del esqueleto (tipo combinado y multiuniones).

En solución acuosa, los PA se auto asocian formando agregados, donde los grupos hidrófobos constituyen el núcleo del agregado y los grupos hidrófilos actúan como barreras protectoras, evitando el contacto de los hidrófobos con el disolvente acuoso. El radio típico de los agregados es de 10 a $40 \mathrm{~nm}$, incluyendo al núcleo hidrocarbonado de $4 \mathrm{~nm}$. Mientras que el ácido desoxirribonucleico (ADN) es una molécula cargada, semiflexible lineal, compuesta de doble cadena que contiene la información genética para el desarrollo biológico de las células vivas. En disolución acuosa al incrementarse la concentración de ADN y por encima de la concentración crítica $\phi^{*}$, las moléculas se entrelazan presentando el sistema comportamientos viscoelásticos más complejos. Una caracterización estructural y reológica del ADN en disolución acuosa o en salmuera es esencial para aplicaciones del tipo génica [5]. El comportamiento viscoelástico complejo que presentan los PA y el ADN en disolvente acuoso es debido, en gran parte, a la estructuración en redes temporales tridimensionales conformada por las uniones entre agregados. Hoy en día y a pesar de la gran cantidad de estudios llevados a cabo en los sistemas de redes termporales tridimensionales de PA y de ADN, aún no, se ha determinado el origen de la viscoelasticidad en terminos estructurales.

En este trabajo se presenta el comportamiento viscoelástico de dos sistemas que forman geles físicos transitorios: ácido desoxirribonucleicos (ADN) disuelto en buffer y el polímero asociativo del tipo multiuniones en el régimen semidiluido no entrelazado en disolución acuosa, mediante las técnicas experimentales de reología mecánica y microreología, en función de la concentración de PAy ADN respectivamente y del tamaño de las partículas de prueba.

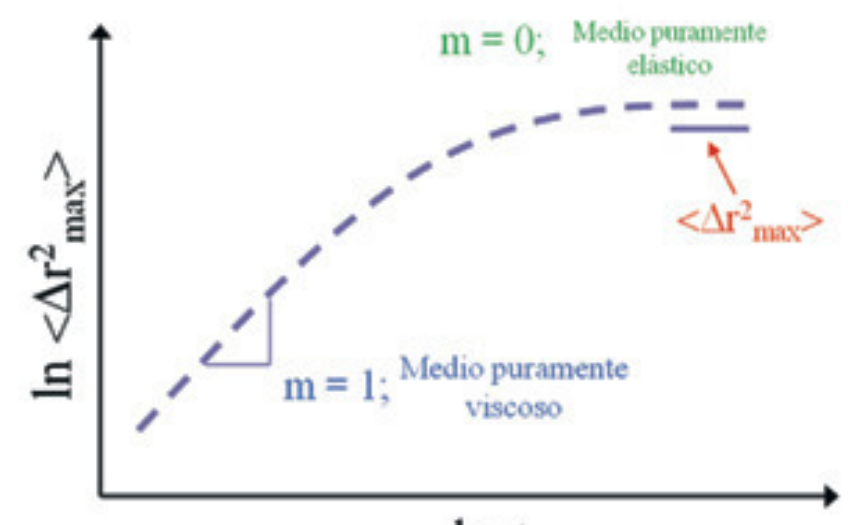

$\ln t$

Figura 3. Esquema del desplazamiento cuadrático medio de sistemas de partículas coloidales dispersas en sistemas viscoelásticos. La pendiente $m=1$ muestra el comportamiento típico de partículas difundiéndose en un medio puramente viscoso, y $m=0$ corresponde a un medio puramente elástico.

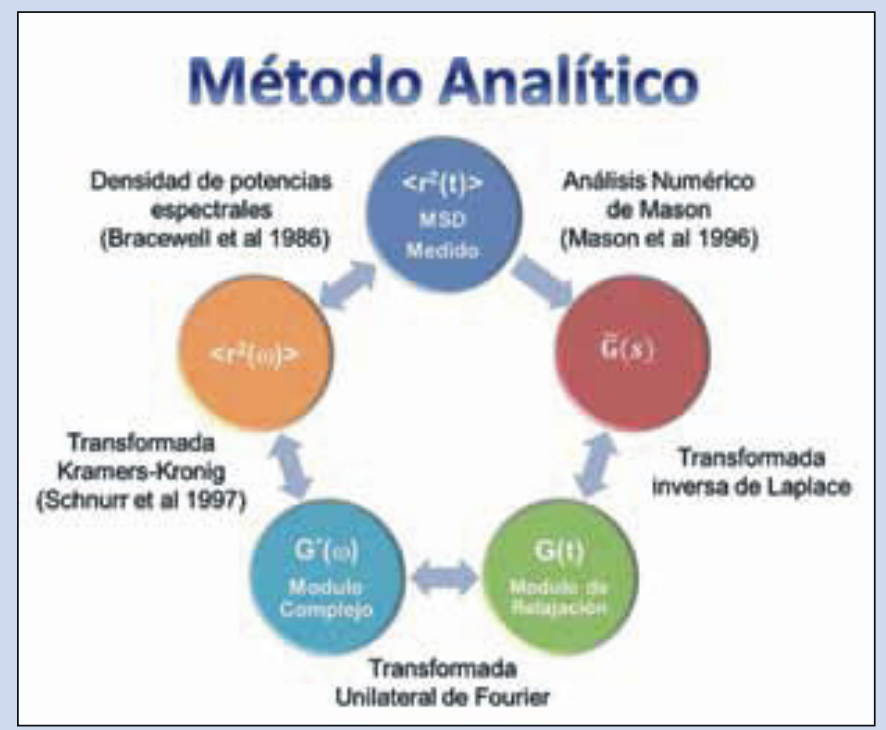

Figura 4. Esquema de obtención de los módulos viscoelásticos a partir del desplazamiento cuadrático medio. 


\section{METODOLOGÍA EXPERIMENTAL}

\subsection{Preparación de muestras de Polímeros Asociativos}

El polímero asociativo de tipo multiunión (PAM-co-DOAM) utilizado en este trabajo es una poliacrilamida (PAM) modificada hidrófobamente, con un peso molecular de $M_{w}=$ $209,000 \mathrm{~g} / \mathrm{mol}$ y un índice de polidispersidad, $M_{w} / M_{n}=2.5$, con pequeñas cantidades de $\mathrm{N}, \mathrm{N}$-dioctilacrilamida (DOAM) ( $1 \%$ molar), y fue obtenido mediante polimerización por radicales libres en solución.

\subsection{Preparación de muestras de $A D N$}

Todas las muestras tanto de PA, como de ADN fueron preparadas pesando soluto y solvente. En el caso particular de ADN fueron preparadas pesando ADN de timo de ternera con 13,000 pares de bases (Sigma-Aldrich) en buffer acuoso (10 mM Tris-HCl y $0.1 \mathrm{mM}$ EDTA) con un $\mathrm{pH}$ ajustado a 8.0 en viales de $20 \mathrm{~mL}$.

\subsection{Muestras con partículas coloidales}

Para el estudio microreológico se realizaron muestras de PA y ADN con partículas esféricas trazadoras de poliestireno dispersas en las soluciones. Las partículas utilizadas son de la marca DukeScientific $\odot$ con un diámetro de $0.3 \mu \mathrm{m}$ y una concentración en la muestra de $0.002 \%$ en peso. Tanto el tamaño como la concentración de la partícula en solución, se escogió pensando en evitar contribuciones reológicas debido al movimiento traslacional de las partículas a nivel microscópico y evitar que las soluciones se tornaran turbias por efecto de la alta concentración, provocando dispersión múltiple.

\subsection{Reología lineal oscilatoria}

Las mediciones de reología lineal oscilatoria fueron llevadas a cabo utilizando un reómetro de la marca Anton-Paar de esfuerzo controlado, modelo MCR300. La geometría utilizada fue de cono-plato $(50 \mathrm{~mm}$ de diámetro y un ángulo de contacto $\left.\alpha=0.98^{\circ}\right)$. La disminución de la evaporación del disolvente de las muestras se redujo utilizando una cubierta húmeda sobre la geometría. El control de temperatura se realizó utilizando un sistema tipo Peltier.

\subsection{Dispersión Dinámica de Luz}

Las mediciones de dispersión dinámica de luz se realizaron con un dispersor ALV/DLS/SLS-5000 ALV GmbH; Langen, Alemania, y utilizando un láser Helio-Neón con una longitud de onda de $632.8 \mathrm{~nm}$. La función de autocorrelación intensidad normalizada en tiempo real es definida

$$
g_{2}(k, t)=\langle I(k, 0) I(k, t)\rangle /\langle I(k, 0)\rangle^{2}
$$

donde $g_{2}(k, t)$ es la función de correlación de intensidades, $\langle\ldots\rangle$ denota el promedio temporal, y $k=(4 \pi n / \lambda) \operatorname{sen}(\theta / 2)$, es el vector de onda dispersada. En este trabajo, el índice de refracción fluctúo alrededor de 1.338, y el ángulo de dispersión se mantuvo fijo a $90^{\circ}$. La función de correlación experimental está relacionada con la función de autocorrelación normalizada por medio de la ecuación de Siegert $g_{2}(t)=1+\beta\left|g_{1}(t)\right|^{2}$, donde $\beta$ es un factor de corrección experimental que depende del arreglo óptico del equipo y es próximo a 1. Suponiendo una distribución gaussiana, el desplazamiento cuadrático medio $\left\langle\Delta r^{2}(t)\right\rangle$ de las partículas puede ser calculado a partir de la función de correlación de campo electromagnético $g_{1}(t)=\exp \left[-\left(k^{2} / 6\right)\left\langle\Delta r^{2}(t)\right\rangle\right]$. Para el caso de partículas trazadoras esféricas, rígidas y monodispersas, la dinámica traslacional depende del coeficiente de difusión $D_{0}$, que es obtenido a partir del coeficiente de difusión StokesEinstein dado por la ecuación $D_{0}=\frac{k_{B} T}{6 \pi a \eta}$, donde $k_{B} T$ es la energía térmica del sistema, $a$ es el radio de la partícula y $\eta$ la viscosidad del medio circundante a las partículas de prueba. La ecuación de Stokes-Einstein se generaliza al considerar que el medio presenta una viscosidad compleja $\eta *(\omega)$.

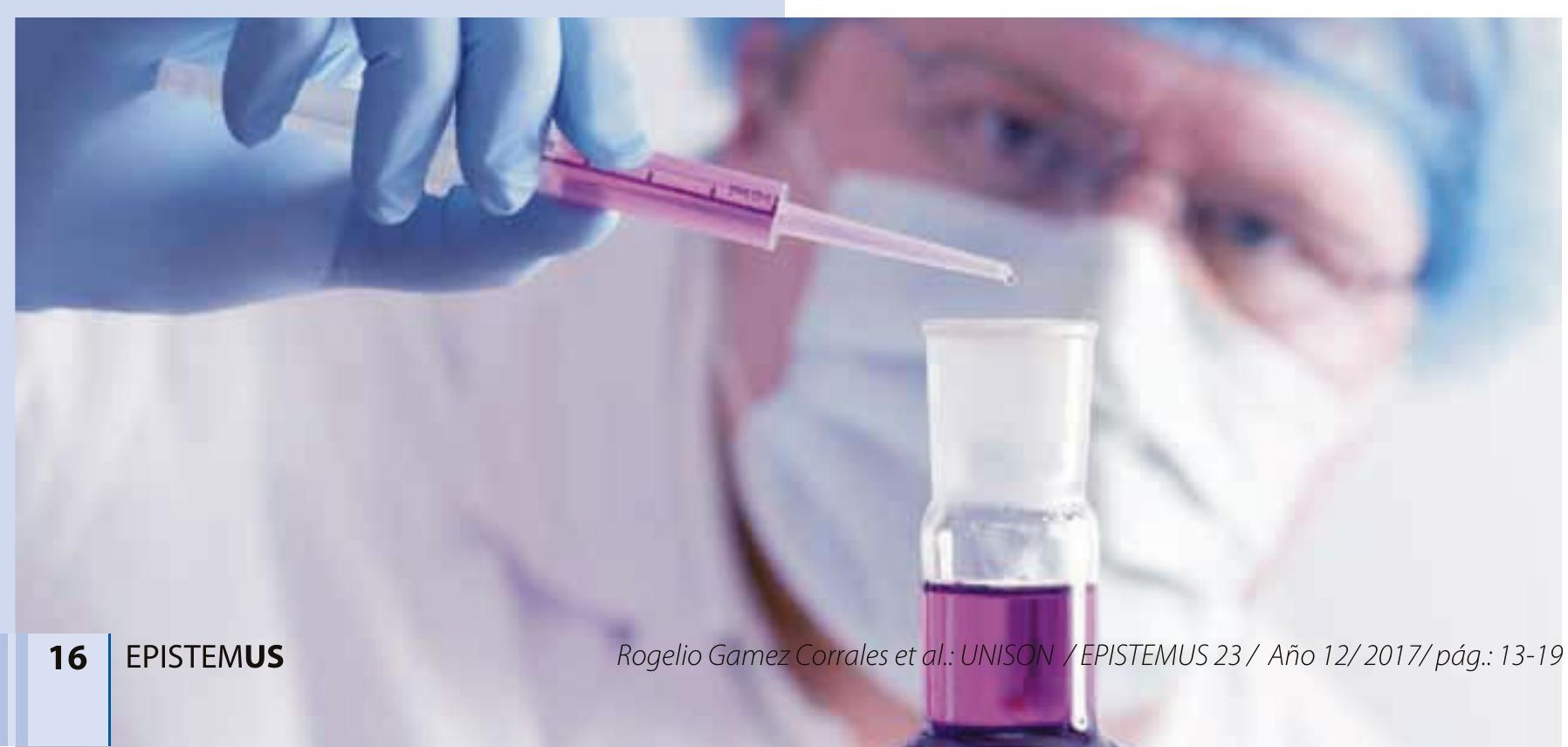




\subsection{Microreología}

La microreología en la práctica se basa en agregar partículas coloidales al sistema que se está estudiando. En la Figura 1 se muestra esquemáticamente el procedimiento utilizado para el caso particular de PA y del ADN en disolventes acuosos. Es indispensable que la interacción entre la matriz viscoelástica y las partículas coloidales sea muy débil o nula, con la finalidad de no modificar el comportamiento reológico de la matriz. Así, las partículas coloidales tienden a moverse dentro de la matriz compleja, por efecto de las fluctuaciones térmicas del entorno, haciendo posible utilizar la ecuación de Stokes-Einstein para determinar la viscoelasticidad del medio en el cual se encuentran dispersas. El movimiento browniano de las partículas es cuantificado por medio del desplazamiento cuadrático medio, $\left\langle\Delta r^{2}(t)>\right.$ que realizan las partículas. En el caso de medios viscosos $<\Delta r^{2}(t)>$ mantiene una tendencia lineal con el tiempo, con pendiente 1 (ver Figura 3), mientras que en el caso de un medio sólido el valor es 0.

La obtención de las propiedades elásticas y/o viscosas del medio puede ser calculada utilizando varios métodos matemáticos, como se muestra en la Figura 4. La ruta utilizada en este trabajo para determinar la viscoelasticidad de las disoluciones de PA o de ADN por medio de microreología, es la desarrollada por T.G. Masonet al., basada en la utilización de una aproximación analítica [6]. El método analítico de Mason, hace una relación entre los módulos de almacenamiento de energía $G^{\prime}(\omega)$, y de pérdida $G^{\prime \prime}(\omega)$, con el desplazamiento cuadrático medio de las partículas de prueba:

$$
G^{\prime}(\omega)=\left|G^{*}(\omega)\right| \cos \left[\frac{\pi \alpha(\omega)}{2}\right] \quad y \quad G^{\prime \prime}(\omega)=\left|G^{*}(\omega)\right| \sin \left[\frac{\pi \alpha(\omega)}{2}\right]
$$

con,

$$
\left|G^{*}(\omega)\right| \approx \frac{k_{B} T}{\pi a<\Delta r^{2}(1 / \omega)>\left.\Gamma[1+\alpha(\omega)]\right|_{t=1 / \omega}}
$$

donde, $\alpha(\omega)$ es el exponente de la ley de potencias que describe la pendiente logarítmica del $\left\langle\Delta r^{2}(t)\right\rangle$ a $t=1 / \omega \omega$, es decir:

$$
\alpha(\omega)=\left.\frac{d \ln <\Delta r^{2}(t)>}{d \ln t}\right|_{t=1 / \omega}
$$

El módulo complejo puede relacionarse con la viscosidad compleja a través de la expresión $\eta^{*}(\omega)=G^{*}(\omega) / \omega$

\subsection{Resultados y discusiones}

La Figura 5 muestra el desplazamiento cuadrático medio $\left\langle\Delta r^{2}(t)\right\rangle$ de las disoluciones de PA y ADN respectivamente. El tamaño y concentración de partículas coloidales se mantuvo constante en ambas mediciones $(d=0.3 \mu m ; \phi=0.002 \% p / p)$, la concentración de soluto varió de $\phi=3 \% \mathrm{p} / \mathrm{p}$ paraladisolución dePA y $\phi=2 \mathrm{mg} / \mathrm{mL}$ para la disolución de $A D N$, ambas mediciones se realizaron a $30^{\circ} \mathrm{C}$. En la Figura 5 se observa que predominan dos regímenes temporales cercano a los $2 \mathrm{~ms}$ para ambos sistemas. En el régimen a tiempos cortos, la disolución de PA muestra un comportamiento subdifusivo con una pendiente de 0.3 , mientras que en el caso de disoluciones de $A D N$, el comportamiento dominante es difusivo (valor de pendiente 1). A tiempos largos, las partículas de prueba dispersas en la disolución de PA se difunden con una

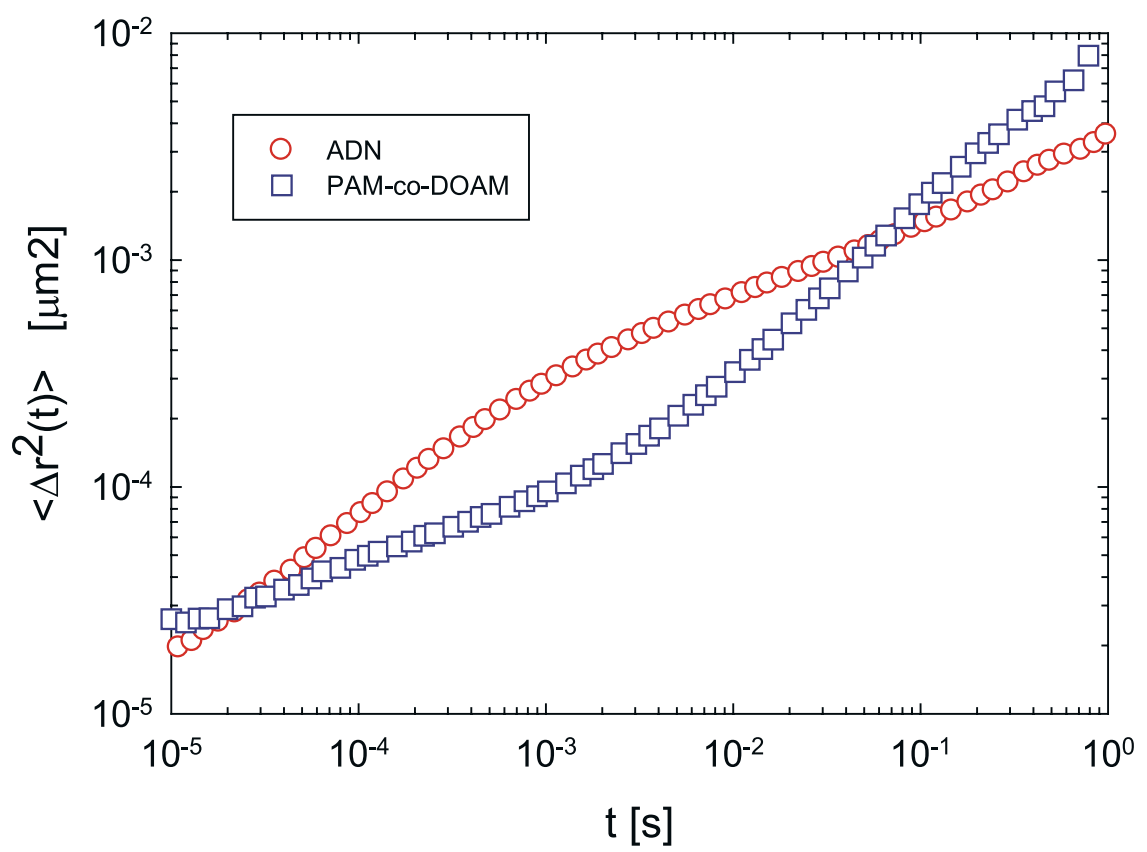

Figura 5.- Desplazamiento cuadrático medio de ADN (círculos vacíos) en disolución a una concentración $2 \mathrm{mg} / \mathrm{mL}$ con partículas coloidales de $0.3 \mu \mathrm{m}$ a una concentración de $0.002 \%$ en peso. Los cuadros vacíos corresponden a disoluciones acuosas de PA multiuniones a $\phi=3 \%$ en peso con partículas coloidales (a $0.3 \mu \mathrm{m}$ a $0.002 \%$ en peso) y $\mathrm{T}=30^{\circ} \mathrm{C}$. 
pendiente de 0.6. En el caso de las disoluciones de ADN, se observa que en el régimen a tiempos largos predomina un movimiento subdifusivo con una pendiente de 0.3. Estos comportamientos corresponden a estados intermedios entre el estado viscoso y el elástico reflejando el ambiente complejo en el que se difunden las partículas coloidales, formadas por las redes temporales de ambos sistemas.

Cabe mencionar que todas las concentraciones tanto de los PA como del ADN, utilizados en este trabajo se encuentran en el régimen semidiluido no entrelazado [7]. Los valores de concentraciones de soluto, fueron elegidos debido a que la técnica de microreología utilizando DLS es muy precisa en este régimen de concentración, debido a que el medio viscoelástico es homogéneo.

En la Figura 6 se muestran los módulos elástico $G^{\prime}(\omega)$ y viscoso $G^{\prime \prime}(\omega)$ en función de la frecuencia $\left(0.1 s^{-1} \leq \omega \leq 100 s^{-1}\right)$ de disoluciones acuosas del PA a una concentración de $\phi=3 \% p / p$ y a una temperatura de $30^{\circ} \mathrm{C}$. La concentración de partículas se mantuvo constante $(\phi=0.002 \% p / p)$. Estas mediciones son obtenidas utilizando reología oscilatoria, con una deformación en el régimen lineal, y complementadas con mediciones de microreología utilizando el método analítico de Mason et al. [8]. En estas mediciones, la variación en el diámetro de las partículas empleadas en este trabajo no genera cambios significativos en los módulos viscoelásticos respecto a la solución del PA o de ADN sin partículas trazadoras $[9,10]$.
Así mismo, se observa un comportamiento viscoelástico, donde el modulo viscoso predomina sobre el módulo elástico. Ambos módulos presentan un comportamiento en leyes de potencias próximo a 1, la extrapolación de estos módulos a frecuencias superiores a $100 \mathrm{~s}^{-1}$ da como resultado un tiempo de relajación característico del sistema $\lambda \approx 0.16 \mathrm{~ms}$. Por otra parte, se observa un buen traslape entre las mediciones de bulto o reología mecánica con las realizadas con la técnica de microreología permitiendo asociar mecanismos responsables de los comportamientos viscoelásticos de los sistemas estudiados, con sus comportamientos macroscópicos.

Los módulos viscoelásticos muestran una tendencia compleja, caracterizado por una desviación de las pendientes logarítmicas de un comportamiento en un medio puramente viscoelástico. De tal manera que no puede ser interpretado por un modelo mecánico ideal como el de Maxwell, siendo necesario utilizar teorías de polímeros más complejas.

Los comportamientos viscoelásticos de estos dos sistemas de PA y ADN, muestran, respectivamente, leyes de potencias correspondientes a los modos de respiración de Rouse (a frecuencias altas), donde ambos módulos viscoelásticos $G^{\prime}(\omega)$ y viscoso $G^{\prime \prime}(\omega)$ presentan una pendiente logarítmica de $1 / 2$.

El modelo de Rouse es formulado al considerar un conjunto de resortes acoplados de manera lineal, los
Figura 6. Microreología (símbolos abiertos) versus reología mecánica (símbolos cerrados) de PA multiuniones en soluciones a una concentración en peso del $3 \%$ con partículas de $0.3 \mu \mathrm{m}$ a $0.002 \%$ de concentración y a $\mathrm{T}=30^{\circ} \mathrm{C}$.

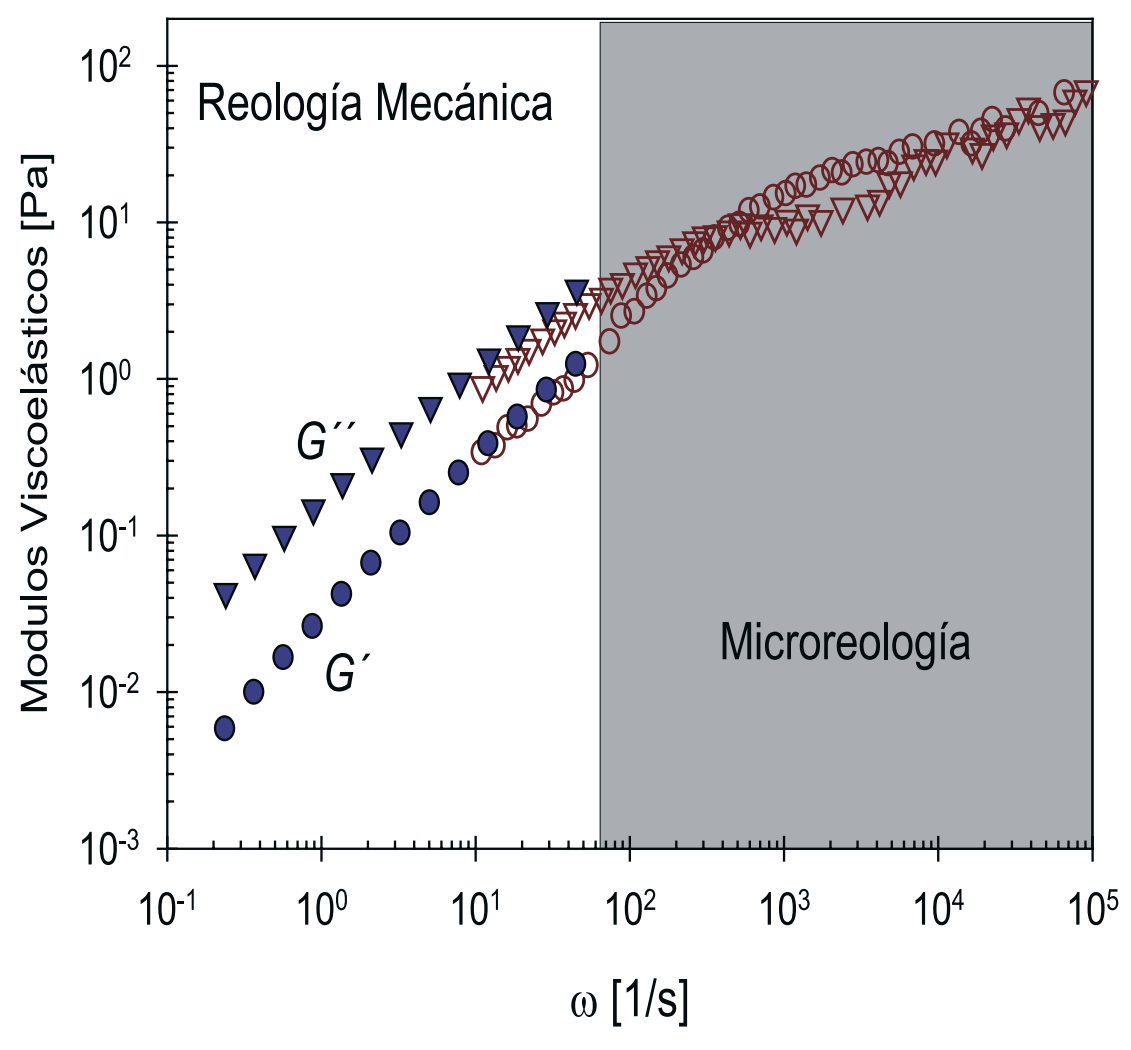

Rogelio Gamez Corrales et al.: UNISON / EPISTEMUS 23 / Año 12/2017/pág.: 13-19 


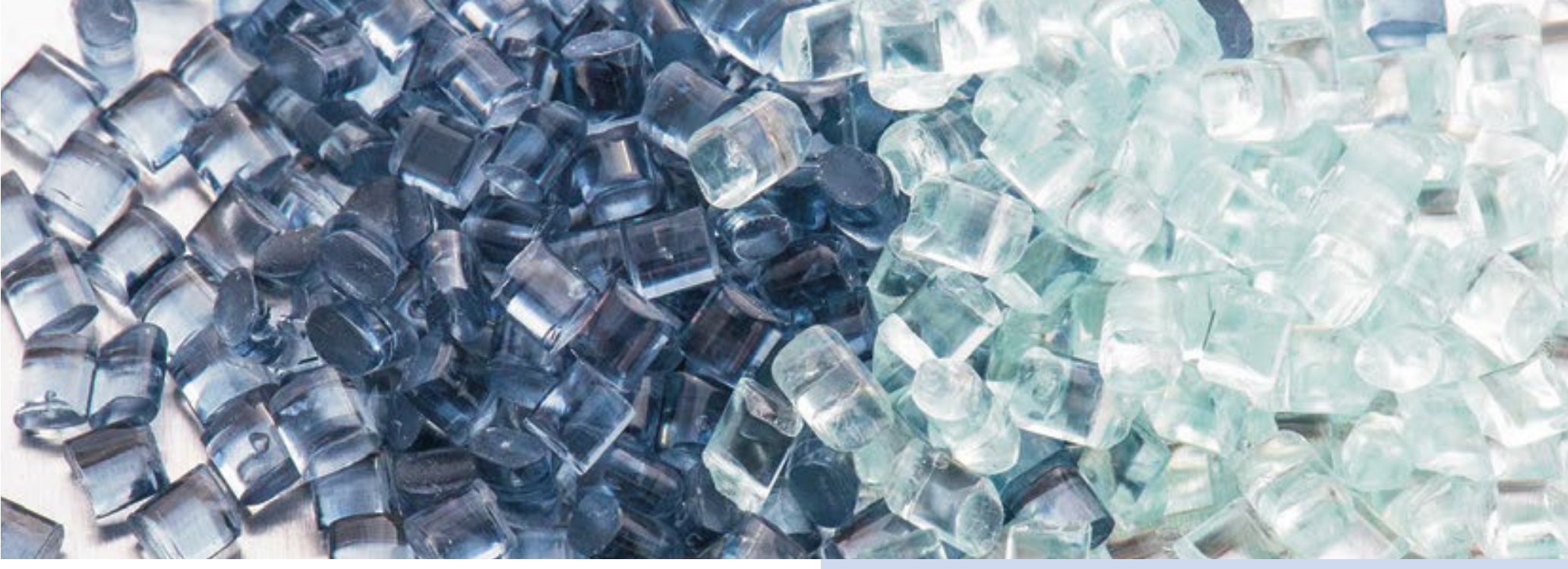

cuales están caracterizados por una masa puntual y una constante de resorte. Este modelo es válido en el caso de sistemas poliméricos en el régimen de concentraciones diluidas. Sin embargo, en sistemas complejos, no siempre representa totalmente el comportamiento real. Como se puede apreciar, una clara desviación de este comportamiento típico del modelo de Rouse a frecuencias intermedias, donde en dos ocasiones se entrecruzan los valores de los módulos viscoelásticos. Las frecuencias de entrecruzamiento denotan transiciones entre los regímenes, dominado por una dinámica de rompimiento de agregados y el dominado por Rouse.

\section{CONCLUSIONES}

En este trabajo se realizó el estudio de las propiedades viscoelásticas de dos sistemas de fluidos complejos, los cuales forman redes temporales a escala nanométrica. Un sistema de PA en disolución acuosa, caracterizado por formar estructuras tipo flor debido a interacciones hidrofóbicas, mientras que el caso de disoluciones de ADN en buffer se caracteriza por formar redes complejas por interacciones de puentes de hidrógeno [10]. La técnica de reología mecánica fue utilizada para estudiar las propiedades viscoelásticas de bulto de ambos sistemas. Mientras que para las propiedades viscoelásticas a nivel microscópico, se empleó la técnica de dispersión dinámica de luz para medir la microreología de los sistemas estudiados. La microreología fue elucidada a través de la difusión de partículas coloidales inmersas en las disoluciones poliméricas, transformando el espacio de tiempos al espacio de frecuencias utilizando el método analítico de Mason et al. [8]. De esta manera se amplió el dominio de validez de los módulos viscoelásticos $G^{\prime}(\omega)$ y $G^{\prime \prime}(\omega)$; lográndose obtener un buen traslape entre los comportamientos viscoelásticos macroscópicos y microscópicos en cada uno de los sistemas. Los modos normales de vibración fueron interpretados en base al modelo de Rouse.

\section{AGRADECIMIENTOS}

Los autores agradecen al Dr. Miguel A. Valdés Covarrubias por permitir realizar las mediciones de dispersión dinámica de luz en el laboratorio de Fluidos Complejos del Departamento de Física de la Universidad de Sonora. Y al CONACYT por proporcionar el apoyo económico al estudiante Emmanuel Robles-Avila para la realización de sus estudios de Maestría en Ciencia de Materiales en el Departamento de Investigación en Polímeros y Materiales de la Universidad de Sonora.

\section{REFERENCIAS}

1) Waigh, T.A., Microrheology of complex fluids, in Reports on progress in physics2005. p. 685-742.

2) MacKintosh, F.C. and C.F. Schmidt, Microrheology. Current Opinion in Colloid \& Interface Science, 1999. 4: p. 300-307.

3) Lu, Q. and M.J. Solomon, Probe size effecs on the microrheology of associating polymer solutions. Phys. Rev. E, 2002. 66: p. 061504.

4) Mason, T.G., et al., Particle tracking microrheology of complex fluids. Phys. Rev. Lett., 1997. 79(17): p. 3282-3285.

5) Gardlik, R., et al., Vector and delivery systems in gne therapy. Med. Sci. Monit., 2005. 11(4): p. RA110-21.

6) Mason, T.G., Estimating the viscoelastic moduli of complex fluids using the generalized Stokes-Einstein equation. Rheol. Acta, 2000. 39: p. 371-378.

7) Jiménez-Regalado, E.J., et al., Study of three different families of water soluble copolymers: synthesis, characterization and viscoelastic bahavior of semidilute solutions of polymers prepared by solution polymerization. Polymer, 2004. 45: p. 1993-2000.

8) Mason, T.G., Estimating the viscoelastic moduli of complex fluids using the generalized Stokes-Einstein equation. Rheological acta, 2000. 39: p. 371-378.

9) Robles, E., et al., Microreología de polímeros asociativos multiuniones en solución acuosa.Revista Mexicana de Física, 2010. 56(2): p. 106.

10) Carvajal, F., et al., Microrheology study of semidiluted deoxyribonucleic acid solutions. Materials Research Society, 2010. 1277: p. 34. 